\section{Can this model be scaled up?}

The model described above depends on one supervising psychiatrist covering up to six refugee camps together with the surrounding villages, equivalent to a general population of about 100000 . Each camp has clinics and basic therapeutic activities run by a specialist community mental health nurse supported by trained volunteers and other health workers. But the important question in relation to sustainability is whether, without international input, such a model can be replicated across all refugee camps in Chad, or be scaled up to run nationally. Sadly, the answer is probably not, mainly because of the lack of available local psychiatrists, although specialist mental health nurses are also in short supply. The Mental Health Atlas published by the World Health Organization (2005) describes Chad as having no mental health policy, and 0.01 psychiatrists per 100000 people. The country has a population of 10.3 million. A more recent estimate of psychiatrists in a number of other African counties (Jenkins et al, 2010) estimates an average of 0.33 per 100000 people, but does not include Chad. It is hard to know how accurate these figures are, but they confirm my impression that, compared with many other African countries, Chad is particularly short of trained mental health staff. So, without the prospect of local recruitment, the existing programme is dependent on international aid for psychiatrist input and, given the demands on donors elsewhere, this is unlikely to be scaled up in the future, and may indeed be phased out at some point.

In the long term, therefore, true sustainability of the existing programme, and further development of services across the country, could be achieved only through big institutional infrastructure projects such as supporting the Chadian government to design a funded mental health policy and strategy, and by developing national training programmes for mental health professionals.

\section{Lasting memories}

Imprinted most lastingly on my memory is the dignity with which those who have lost everything conduct themselves. This was apparent in the way families presented at clinic, squeezed on to rough benches, patiently and gracefully waiting their turn, often dressed in colours and tribal designs that echoed their rich heritage as descendants of a powerful 19th-century North African kingdom. And patients almost always attended with their family. So the strategy of keeping families and local communities together after the chaotic mass exodus from Darfur appears to have worked, thereby protecting the all-important social capital and natural resilience associated with relationships that is so crucial for mental well-being in the aftermath of trauma (Pfefferbaum, 2008)

\section{References}

Central Intelligence Agency (2009) The World Fact Book. Chad data on refugees and internally displaced persons. Available at https://www. cia.gov/library/publications/the-world-factbook/geos/cd.html (accessed September 2009).

Danvers, K., Sivayokan, S., Somasundaram D. J., et al (2006) Ten months on: qualitative assessment of psychosocial issues in northern Sri Lanka following the tsunami. International Psychiatry, 3(3), 5-8.

Flint, J. \& De Waal, A. (2008) Darfur: A New History of a Long War. Zed Books.

Jenkins, R., Gureje, O., Mullen, P., et al (2010) International migration of psychiatrists. PLOS One. Available at http://dx.plos.org/10.1371/ journal.pone.0009049 (accessed September 2009).

Makhdum, M. A. \& Javed, A. (2006) Earthquake in Pakistan and Kashmir: suggested plan for psychological trauma relief work. International Psychiatry, 3(1), 16-18.

Pfefferbaum, R. L. (2008) Factors in the development of community resilience to disasters. In Intervention and Resilience After Mass Trauma (eds M. Blumenfield \& R. J. Ursano), pp. 49-68. Cambridge University Press.

World Health Organization (2005) Chad. In Mental Health Atlas, pp. 127-128. WHO.

\title{
Getting your paper published
}

\section{Patricia Casey}

Editor, The Psychiatrist, and Professor of Psychiatry, Mater Misericordiae University Hospital, Dublin, Ireland, email apsych@mater.ie

단 mbarking on a research project is always exciting, although a large number are not completed. One study of pharmacotherapy projects submitted to a research ethics committee found that after 5 years more than a quarter had not been completed and almost a sixth were considered unpublishable (Winther \& Hole, 1997).

Achieving publication is the aim of most doctors. The primary motivation for this varies from a wish to enhance job prospects to boosting one's ego and a desire to see one's name in print. The most noble, of course, is a desire to spread knowledge and contribute to the science of our specialty.

The proportion of submitted papers that ultimately succeed in achieving publication varies greatly; journals with a high impact factor have the lowest yield. For The Psychiatrist, the proportion of submitted papers that are published is around $50 \%$, well above the $16.6 \%$ identified in one study of eight high-impact English-language journals (although only five replied to the study questionnaire) (Singh, 2006), a figure that fell to only $4.4 \%$ when papers from low- and 
middle-income countries were examined. This finding of underrepresentation of published output from those outside Europe, Australia and North America has been replicated by others (Patel \& Sumathipala, 2001).

\section{What is a quality paper?}

There is an assumption that once a research project has been completed, it will be worthy of publication in a scientific journal. This is misguided; it is often forgotten that nobody has a 'right' to have their work published. Quality always supersedes ego. Quality refers to the methodology and relevance of the research project itself and also to the way in which a paper is written and presented. The latter is within the control of the writer, since the style of writing and the focus of the paper come from the researcher. It is also an attribute that can be developed as the preparation of the paper progresses. On the other hand, for beginners, the quality of the research project itself is more dependent on the advice and supervision of senior colleagues. These are factors that are often outside the control of the investigator, since they depend on having access to supervisors who understand research methods and who are willing to give their time to trainees.

\section{Choosing the project}

Selecting an area to study and developing a research proposal around this is arguably the most vital aspect of the work to achieve publication. If the methods are not sound or if the study design will not answer the question you want to ask, then publication will be next to impossible.

The primary rule is that if you decide to carry out an original piece of research, you must be properly supervised from the beginning. Where there are difficulties obtaining guidance and supervision locally, the assistance of the nearest academic department will be necessary.

You must prepare a written proposal detailing the background to the study, the aim or hypothesis and the methods. This will keep you focused on the specific question you are asking. You need to obtain ethical approval, since most journals require a statement that this approval has been granted before they will consider your paper for review.

\section{The type of study or paper you want to write}

There are papers and studies about which novice researchers are enthusiastic but which hold little appeal for many journal editors. These include:

o case reports

incomplete audit cycles

O retrospective case-note studies

O descriptive papers without any statistical analysis (e.g. describing the development of services or case series)

O reports of studies with small sample sizes

O narrative reviews

O reports of qualitative studies

reports of pilot studies.
Nonetheless, some journals will accept these papers, especially journals that are new to the market. Others will accept them under certain exceptional circumstances. And there are some established journals dedicated to these topics, such as the Journal of Medical Case Reports.

Studies that are described as 'qualitative' often do not adhere to the methods required of such investigations and are nothing more that descriptions of conversations with a small sample of people whose comments are extracted and presented in an unstructured style. Before carrying out a qualitative study, you should check with an expert in the field to ensure appropriate methods are used and that this approach is appropriate to your investigation.

\section{Choosing the journal}

In your quest for publication it is worth spending some time identifying a suitable journal where your paper may find a home. Journal databases in libraries are a useful source of this information, as are medical librarians. Ultimately, you may have to search the PubMed journal database yourself or even use search engines such as Google. Reading the reference lists at the end of scientific papers in your field is also a useful way of finding new and obscure journals.

In selecting a journal there should be a match between it and your paper, both in the subject matter and in the quality. For example, a paper on eating disorders has a better chance of publication in a journal dealing with that topic than in a general journal. On the other hand, if the methodological quality of your study is questionable, there is little point in seeking publication in a high-impact international journal. Reading papers from your chosen journal will assist in deciding if your paper stands a chance of being considered by the particular journal.

A further consideration is whether there are any other restrictions on what is accepted by the journal. For instance, some do not accept case reports, whereas others do not accept narrative reviews and some journals accept mainly, or only, commissioned reviews.

\section{Writing your paper}

Careful attention should be paid to the house style of the journal, such as the style of referencing, the number of tables and figures allowed and the length required.

The title should be short and to the point. If possible, avoid mentioning a local town or city in the title, since this immediately restricts the likely interest and gives the impression of parochialism. The abstract should contain only basic data and should be an accurate summary of the content of your paper.

The length of the section dealing with the background to the study varies from journal to journal and you should check this by reading other recent papers in your chosen publication. This section should conclude with the aims or hypotheses being tested.

When presenting the results, avoid duplicating what is in the tables with detailed descriptions of these in the text also. Simply point out the key findings of the tables in the text. Many editors now prefer confidence intervals to $P$ values and 
it is important to ascertain, in consultation with your statistician, that the correct statistics have been used.

The discussion should not repeat the results. Instead, the focus should be on dealing with the implications of your findings as well as on possible explanations of the discrepancies between the results of your study and those of others. Always mention the limitations of your study.

\section{Comprehensibility}

The ease with which the paper can be understood is highly important. Even with powerful data, if it is difficult to read or to understand the report, then reviewers are likely to be critical. Faced with a turgid and dense style a reviewer may simply reject a paper or recommend a complete rewrite. To assess the comprehensibility of your paper it is advisable to ask colleagues to read it and to feed back to you honestly. If the language of your chosen journal is not your mother tongue, the opinion of a person fluent in the spoken and grammatical aspects of that language should be sought.

One reason for difficulties understanding papers is that too much information is presented. Simply because a statistical test has been carried out does not mean that it needs to be included in the paper. Use only the data that are relevant to testing your specific hypothesis.

\section{Relevance}

There is an understandable tendency among novice researchers to carry out studies in single services, owing to the convenience of the sample. This may limit the generalisability of the findings. On the other hand, it may be of immense local interest, so consideration should be then given to publishing it in a local journal rather than an international one.

\section{Scientific merits}

The most important aspect with regard to achieving publication is the quality of the study itself. Ask yourself whether the sample size was large enough, and whether a specific hypothesis was identified and tested. Were the correct statistics applied and were the interpretations of these accurate? Some papers may be descriptive only and not use any statistics, but these are less favoured by most journals.

If you write a narrative review it must be of exceptionally high quality to achieve publication. It must contain information that is not accessible in major textbooks and must show a depth of knowledge that only an expert can have. Few journals, except those that commission them, now accept narrative reviews, opting instead for systematic reviews.

\section{Conclusion}

Getting published in a peer-reviewed journal is difficult and requires a significant amount of time and effort, labour that begins the day you conceive of your study. Publication is not inevitable but it is immensely rewarding when it comes to fruition. It also carries with it the possibility that you will be invited to review papers written by that journal. The key to success lies in planning your project carefully at the outset and relentlessly focusing on the quality of your work at each stage of this long process. From the day you decide to begin your study until you receive the letter confirming the acceptance of your paper you may be 4 years older but the wait will have been worth it.

\section{References}

Patel, V. \& Sumathipala, A. (2001) International representation in psychiatric literature: survey of six leading journals. British Journal of Psychiatry, 178, 406-409.

Singh, D. (2006) Publication bias - a reason for the decreased research output in developing countries. South African Psychiatric Review, 9, 153-155.

Winther, F. O. \& Hole, O. P. (1997) Scientific quality of clinical research: an analysis of 40 research projects in pharmacology/pharmacotherapy. European Journal of Clinical Pharmacology, 51, 351-354.
I made an agreement of peaceful coexistence with time: Neither he pursues me, nor I run from him, one day we will find each other.
It may seem a strange principle to enunciate as the very first requirement in a Hospital that it should do the sick no harm.

Florence Nightingale, English nurse, Notes on Hospitals (1863 edn), preface 